\title{
Correction to: Blalock-Taussig Shunt Size: Should it be Based on Body Weight or Target Branch Pulmonary Artery Size?
}

\author{
Emrah Şişli ${ }^{1,5}$ (1) Osman Nuri Tuncer ${ }^{1} \cdot$ Suat Şenkayaa ${ }^{2} \cdot$ Eser Doğan $^{3} \cdot$ Hatice Şahin $^{4} \cdot$ Mehmet Fatih Ayık $^{1}$. \\ Yüksel Atay ${ }^{1}$
}

Published online: 10 September 2018

(c) Springer Science+Business Media, LLC, part of Springer Nature 2018

\section{Correction to: Pediatric Cardiology} https://doi.org/10.1007/s00246-018-1958-9

The original version of the article unfortunately contained an alignment error in Table 1.The correct version of Table 1 is given below.

The original article has been corrected.

The original article can be found online at https://doi.org/10.1007/ s00246-018-1958-9.

Emrah Şişli

dresisli@gmail.com

1 Departments of Pediatric Cardiovascular Surgery, Ege University Faculty of Medicine, Izmir, Turkey

2 Cardiovascular Surgery, Ege University Faculty of Medicine, Izmir, Turkey

3 Pediatric Cardiology, Ege University Faculty of Medicine, Izmir, Turkey

4 Medical Education, Ege University Faculty of Medicine, Izmir, Turkey

5 Section of Pediatric Cardiovascular Surgery, Department of Cardiovasular Surgery, Ege University Faculty of Medicine, Kazım Dirik District, Üniversite Street, 35140 Bornova, Izmir, Turkey 
Table 1 Demographic and clinical characteristics of the patients

\begin{tabular}{|c|c|c|c|}
\hline \multirow[t]{2}{*}{ Characteristics } & \multicolumn{2}{|l|}{ Shunt thrombosis } & \multirow[t]{2}{*}{$p$ value } \\
\hline & $\begin{array}{l}\text { Yes } \\
(n=11)\end{array}$ & $\begin{array}{l}\text { No } \\
(n=69)\end{array}$ & \\
\hline \multicolumn{4}{|l|}{ Demographic } \\
\hline Age (days) & $6(3-24)$ & $4(2-19.5)$ & $0.523^{\mathrm{a}}$ \\
\hline Weight $(\mathrm{kg})$ & $2.6(2-3.1)$ & $3.3(3.0-3.8)$ & $0.004^{\mathrm{a}}$ \\
\hline Weight $<3 \mathrm{~kg}$ & $7(63.6)$ & $14(20.3)$ & $0.008^{\mathrm{c}}$ \\
\hline Male & $5(45.5)$ & $42(60.9)$ & $0.335^{\mathrm{b}}$ \\
\hline \multicolumn{4}{|l|}{ Clinical } \\
\hline Genetic syndrome/extra-cardiac anomaly & $4(36.4)$ & $2(2.9)$ & $0.001^{c}$ \\
\hline Newborn & $10(90.9)$ & $59(85.5)$ & $0.991^{\mathrm{c}}$ \\
\hline Prematurity & $6(54.5)$ & $3(4.3)$ & $<\mathbf{0 . 0 0 1}^{\mathrm{c}}$ \\
\hline Right pulmonary artery size (mm) & $3(3-3)$ & $4(4-5)$ & $<\mathbf{0 . 0 0 1}^{\mathrm{a}}$ \\
\hline Right pulmonary artery, $z$-value & $-1.97(-2.62$ to -0.82$)$ & $-0.06(-0.78$ to 0.54$)$ & $\mathbf{0 . 0 0 1}^{\mathrm{a}}$ \\
\hline Left pulmonary artery size (mm) & $4(3-4)$ & $5(4-5)$ & $<\mathbf{0 . 0 0 1}^{\mathrm{a}}$ \\
\hline Left pulmonary artery, $z$-value & $-0.30(-2.01$ to 0.42$)$ & $0.56(-0.36$ to 0.95$)$ & $\mathbf{0 . 0 0 5}^{\mathrm{a}}$ \\
\hline Pulmonary artery hypoplasia & $9(81.8)$ & $6(8.7)$ & $<\mathbf{0 . 0 0 1}^{\mathrm{c}}$ \\
\hline Single ventricle physiology & $6(54.5)$ & $24(34.8)$ & $0.209^{\mathrm{b}}$ \\
\hline Pulmonary atresia & $10(90.9)$ & $38(55.1)$ & $0.055^{\mathrm{c}}$ \\
\hline \multicolumn{4}{|l|}{ Operative } \\
\hline \multicolumn{4}{|l|}{ Side of mBTS } \\
\hline Left & $4(36.4)$ & $54(78.3)$ & $0.012^{c}$ \\
\hline Right & $7(63.6)$ & $15(21.7)$ & \\
\hline \multicolumn{4}{|l|}{ Approach } \\
\hline Sternotomy & $6(54.5)$ & $14(20.3)$ & $\mathbf{0 . 0 1 5}^{\mathrm{b}}$ \\
\hline Thoracotomy & $5(45.5)$ & $55(79.7)$ & \\
\hline Absolute shunt size (mm) & $3.5(3.0-3.5)$ & $4(3.5-4)$ & $\mathbf{0 . 0 0 1}^{\mathrm{a}}$ \\
\hline \multicolumn{4}{|l|}{ Size of mBTS } \\
\hline $3.0 \mathrm{~mm}$ & $4(36.4)$ & $3(4.4)$ & $0.004^{c}$ \\
\hline $3.5 \mathrm{~mm}$ & $5(45.5)$ & $21(30.4)$ & $0.323^{\mathrm{b}}$ \\
\hline $4.0 \mathrm{~mm}$ & $2(18.2)$ & $45(65.2)$ & $0.009^{c}$ \\
\hline Size of pulmonary artery shunted (mm) & $3(3-4)$ & $5(4-5)$ & $<\mathbf{0 . 0 0 1}^{\mathrm{a}}$ \\
\hline z-value of pulmonary artery shunted & $-0.82(-2.01$ to 0.42$)$ & $0.51(-0.39$ to 0.83$)$ & $0.010^{\mathrm{a}}$ \\
\hline $\mathrm{S} / \mathrm{W}$ ratio & $1.46(0.97-1.67)$ & $1.17(1.01-1.30)$ & $0.049^{\mathrm{a}}$ \\
\hline $\mathrm{S} / \mathrm{PA}$ ratio & $1.0(0.88-1.17)$ & $0.80(0.70-0.88)$ & $0.002^{\mathrm{a}}$ \\
\hline PDA left open & $8(72.7)$ & $51(73.9)$ & $1.0^{\mathrm{c}}$ \\
\hline
\end{tabular}

Bold values indicate statistical significance at $p<0.05$

${ }^{a}$ Mann-Whitney U test

${ }^{\mathrm{b}} \mathrm{Chi}$-square test

${ }^{c}$ Fisher's exact test 